\title{
Long channel for energy deliver (III)
}

\author{
Victor V. Apollonov \\ A. M. Prokhorov General Physics Institute, Moscow, Russia; vapollo@kapella.gpi.ru
}

Received 19 March 2012; revised 25 April 2012; accepted 8 May 2012

\begin{abstract}
At present time the development of new methods of the single-wire long distance energy transfer is of great interest of science. These works are the prolongation and development of Tesla studies in the beginning of XX century. In particular, not so long ago highly effective energy transfer between two resonant circuits, connected by very thin wire $(100 \mathrm{mkm})$ with more than kilometer length had been demonstrated. In these experiments Tesla type transformers with the output voltage about several ten kilovolts and output power up to $10 \mathrm{~kW}$ were used [1]. An effective replacement of wire by laser-plasma channel in that configuration of experiment would be the obvious improvement of demonstrated technology.
\end{abstract}

Keywords: Lightcraft; Impulsar; Conductivity of Dust Plasma; High Power Lasers; Optical Breakdown; Shock Waves

\section{LIGHTNING PROTECTION SYSTEM}

From other side during the last 20 years in many laboratories of the world very interesting studies of effective laser lightning protection system creation had been conducted. On the basis of pulsed laser, long enough laser spark can be created, which is able to connect thunderstorm cloud with the grounded iron core-classical lightning rod. The maximum lengths - $16 \mathrm{~m}$ of such highly controlled by the laser (Figure 1) electrical discharge channel-have been obtained in Russia and in Japan with help of developed and produced in A. M. Prokhorov GPI RAS pulsed $\mathrm{CO}_{2}$ lasers with the energy up to $0.5 \mathrm{~kJ}$.

Later also we have demonstrated much longer laser spark, obtained with the aid of conical optics, which has exposed much more advanced characteristics from the point of view of plasma channel conductivity and very short time delay of its effective start. In our works for the first time the possibility to use as the single wire connecting two resonant circuits by long laser spark, created with the help of conical optics, had been suggested.
At present time among an enormous quantity of different types of lasers for electrical discharges directed by the laser spark wide spectrum of application only two types of lasers are found as the most effective for this particular task: pulsed sub-microsecond $\mathrm{CO}_{2}$ lasers and femtosecond (FS) solid state lasers.

Possibility of one or several parallel highly ionized channels creation, so called filaments, with a characteristic diameter of $100 \mathrm{mkm}$. In the atmosphere along the direction of the laser beam, propagation is the major feature of FS lasers. With estimated value of electron densities of such a filaments (up to $10^{16} 1 / \mathrm{cm}^{3)}$ and with wavelength of the laser in the interval $0.5-1.0 \mathrm{mkm}$, plasma, practically, does not absorb laser emission. In this case the length of channel is determined by the intensity of laser emission and in the case of energy of the FS pulse - of $100 \mathrm{~mJ}$ can reach the length up to $150 \mathrm{~m}$. The possibility of a considerable increase of filaments length is connected with the application of unique super powerful FS laser systems with the energy about $1 \mathrm{~J}$ and even more. Such a system in our days looks like a unique laboratory setup and hardly can be used for commercial and other applications. Another limiting factor is a very high resistance of conducting channel based on FS laser filaments. Characteristic value of resistance of such a channel is on the level of $0.1 \mathrm{Mohm} / \mathrm{m}$ [2].

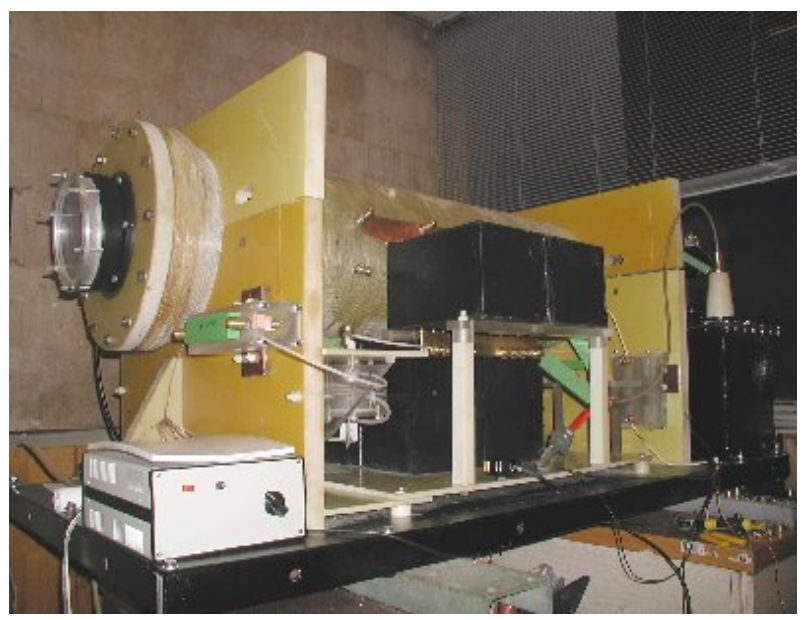

Figure 1. TC-300. Electrical discharge $\mathrm{CO}_{2}$ laser with output$300 \mathrm{~J}$. 
In the case of pulsed $\mathrm{CO}_{2}$ laser use in combination with classical spherical or conical optics the length of spark channel is determined by the parameters the caustics of laser and that its part in which the intensity of emission it exceeds threshold for the optical breakdown of air. The presence of intensity threshold is connected with the special features of the mechanism of plasma formation where the avalanche multiplication of initial "background" electrons in the field of laser emission takes place. A vital difference in the geometry of spark in the case of long pulse and spherical optics from the case with the FS lasers is the fact that the laser spark occupies volume inside the caustic curve from the focal plane to the side of laser, since the laser radiation cannot traverse self created plasma shield. In this case for the super long spark formation important to use $\mathrm{CO}_{2}$ laser pulses with traditional form, i.e., with short (50 - $100 \mathrm{ns)} \mathrm{spike} \mathrm{and}$ long ( $1-3 \mathrm{mks})$ tail [3]. Long tail ensures the additional heating of laser plasma, moving along with the laser beam by expanding to the caustic and, respectively, increasing the length of laser spark. The length of laser spark in several hundred meters has been obtained even in the USSR with the aid $\mathrm{CO}_{2}$ laser with output energyof $5 \mathrm{~kJ}$ and the classical form of the pulse.

However, in the case of conical optics use the possibility of laser plasma channels formation of substantially larger length does appear. According to our estimations the length of such a channels in the case of conical optics in combination with pulse-periodic (P-P) $\mathrm{CO}_{2}$ lasers with an average power of $0.1-1 \mathrm{MW}$ can reach several kilometers. In this case the geometry of spark is completely different and the region "of collapse" is determined by the diameter of the beam on the surface of conical mirror only and by the angle of taper. Spherical optics plasma front moves in the direction of laser beam, i.e., from the surface of the focusing mirror in outside, in this case different spatial domains in the section of laser beam form different sections of plasma cylinder. Therefore the formation of very long laser sparks even with comparatively short lengths of laser pulses is possible. The photo registration of spark showed that it consists of the set of the almost contiguous between themselves elements, on the form resembling "Chinese cap" and located on the identical distance from each other (Figure 2).

Thus, for the experiments conducting the electrical energy transfer between two resonant circuits, situated on the distance not more than $100 \mathrm{~m}$, it is possible to use both types of lasers. At the same time for commercial energy transmission system creation, the powerful high repetition rate $\mathrm{P}-\mathrm{P} \mathrm{CO}_{2}$ lasers appear more promising, since they possess the substantially lower threshold of the optical breakdown in air and technically are more advanced in scaling up of output power to many hundreds of kilowatts.
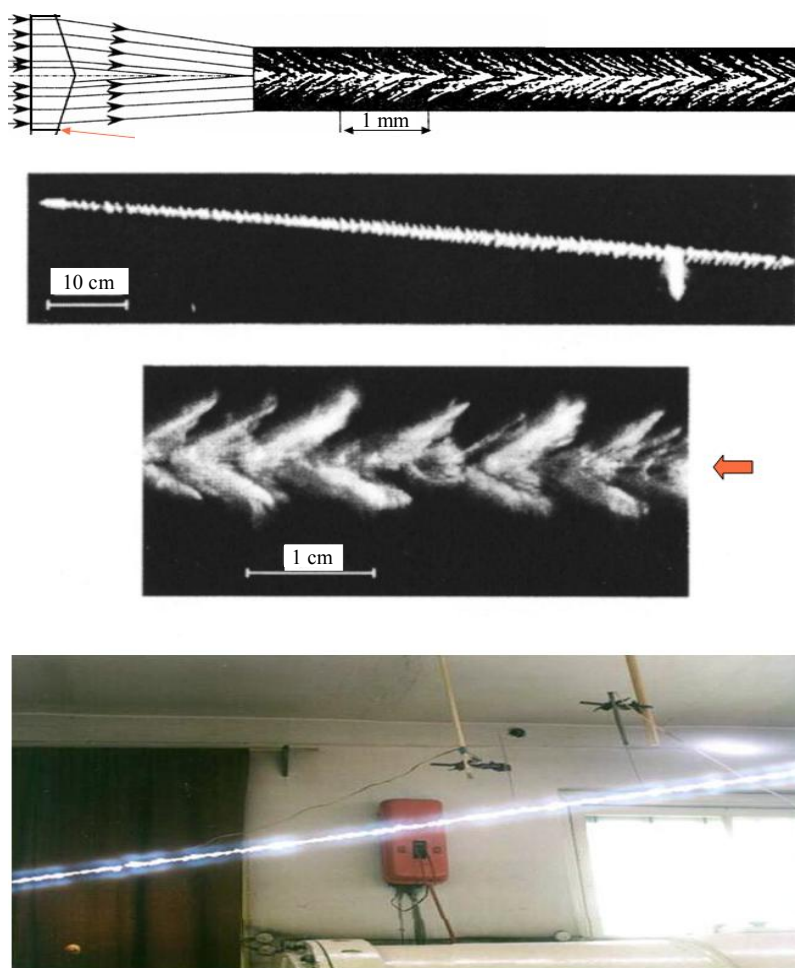

Figure 2. Long conductive channels in atmosphere and their structure.

As has already been spoken above, the geometry of plasma cylinders for two types of laser was completely different: the collection of thin filaments FS laser or cellular single channel with more or less uniform plasmapowerful sub-microsecond $\mathrm{CO}_{2}$. At the present time it is possible to assert that powerful pulse $\mathrm{CO}_{2}$ laser and the mechanism of laser radiation breakdown of gas medium have the decisive advantages over the FS ideology of the energy transfer by long lengths laser plasma channel.

First experiments carried out by our group with pulsed $\mathrm{CO}_{2}$ laser having the traditional form of lasing in the beginning of $90^{\text {th }}$ were very much informative. By potential applied to "the ends" of laser spark (up to $600 \mathrm{~V}$ ) we have showed that the glow of laser plasma remains not more than $10 \mathrm{mks}$, at the same time the conductivity of laser plasma channel does exist considerably longer-up to $100 \mathrm{mks}$. High repetition rate P-P laser emission is capable to solve the tasks of the reproduction of the conducting channel during long period of time and to increase the conducting channel length significantly. The most essential drawback of the described method is the fact that it cannot be directly used in vacuum, which means beyond the limits of the Earth's atmosphere.

New method of the energy transfer and new approach to the transmitting channel formation are directed toward essential simplification of electrical energy transfer methods, at the same time they can be effectively applied to the non-conducting media conditions. Necessary result 
can be achieved due to the "Impulsar" technology.

The energy transfer in this case is achieved because of the fact that in the construction of the focusing system the source of the medium creation in the form of easily ionized substance, which forms the steam-gas medium with low threshold of the breakdown, which is especially important in the case of insufficiently high medium pressure or directly in the vacuum.

\section{NEW APPROACHES FOR CONDUCTIVE CHANNEL CREATION}

The displacement of the optical focusing system in the media and interaction of P-P laser radiation with energy in the focus of optical system sufficient for the breakdown ensures the formation of the continuous currentconducting channel in the air medium due to its ionization, in this case. As calculations show, the frequency band of laser pulses ensures the continuity of the formed current-conducting channel in the appropriate speed range of the moving focusing system, which can be realized in this particular medium. Actually, each pulse of the laser, with the help of focusing system can create a certain extensive region of plasma, which applies to the relatively small section of space in the trajectory of the displacements of that focusing system. If the repetition rate of pulses will follow each other with small frequency, the displacements of the plasma regions after focusing system will be something more similar to the dotted line. However, with higher frequency, for example more than $10 \mathrm{kHz}$, and optimum for the medium speed the displacements of ionization regions will no longer have breaks and the formed by this process conducting channel will be continuous (Figure 3).

However, after entering of moving optical system into much less density layers of the atmosphere and further into the vacuum, the problem of the scarcity of medium appears, which could complete a deficiency in the ionized gas both as the source of conducting medium and the means of shock wave accumulation and transfer to the object of the motion of necessary quantity of energy. For this case the moving optical system must be supplied with the source of medium creation in the environments of laser focus in the form of sublimated under the action

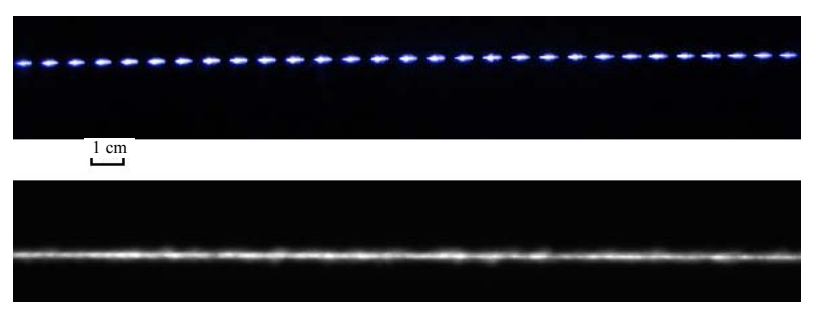

Figure 3. Time controlled evolution of trains in the space for $\mathrm{F}=20 \mathrm{~Hz}, 50 \mathrm{~cm} / \mathrm{s}$ and for $\mathrm{F}=25 \mathrm{kHz}, 250 \mathrm{~cm} / \mathrm{s}$ correspondingly. of incident laser radiation easily ionized substance, containing the nano-particles of metal or substance, which under the action of laser emission ensures the synthesis of such a nano-particle. The presence of easily ionized substance source, which due to the sublimation process under the action of laser emission, makes it possible to form the current-conducting channel in the vacuum ensures the continuity of the canal in this case. Presence of nano-particles of metal in the sublimated substance allows simultaneously with improvement of channel conductivity conditions to increase the value of the specific impulse of thrust of laser jet engine (LJE), containing mobile optical system and reservoir with the sublimated substance, which in the essence with this use has determined the time of reaching the required length of electrical power transmission channel [4].

The introduction of high voltage electrode into the current-conducting channel, formed by the moving optical focusing system, allows, from one side, to stabilize the conducting channel of the required length and direction and from the other side to transfer energy by this channel very effectively. In order to facilitate the breakdown conditions of the medium in the optical system focal point with the appearance of not conducting channel only but high energy shock wave as well, it is expedient to use the easily ionized substances, which form the steam-gas medium as the source of medium creation with low breakdown threshold. The latter fact makes it possible to decrease the requirements for the laser pulse breaking energy value.

The moving focusing system can be executed in the form the conical body or combination of cylinder and cone, inverted by apex to the side of direction of its motion. In the end of moving system, special optics is established, which can be selected from the number of known optical systems, and ensure the focusing of the laser light, which falls down to the optical system at the certain distance from the surface. In the body of moving system, the sublimated under the action of laser emission easily ionized substance is placed, which contains the nanoparticles of metal or substance ensuring their synthesis. As the easily ionized substance wax, paraffin, delrin (high-quality acetate homopolymer) and other polymeric substances can be used. The nano-particles of metal can be of significant dimensions, for example $10-100 \mathrm{~nm}$ and can prepared from the metals such as aluminum, tin, copper, Na, K, Cs, lead and so on. The carbon-containing substances in the combination with the alkali metals can be used as the substance, which makes it possible to synthesize the nano-particles of metal or the metal-composite materials, which possess sufficiently high electrical conductivity. From the literature they are well known - fullerenes on the basis of alkali metals, fullerenes on the basis of coppers, which demonstrate high-tempera- 
ture superconductors properties with tc $>$ of $140 \mathrm{~K}$. For obtaining the nano-crystalline powder the plasma and laser methods of heating can be used. Thus, the nanoparticles of carbides, oxides and nitrides with the aid of the pulse laser heating of metals in the rarefied atmosphere of methane (in the case of carbides), oxygen (in the case of oxides), nitrogen or ammonia (in the case of nitrides) were obtained. The pulse laser evaporation of metals in the atmosphere of inert gas ( $\mathrm{He}$ or Ar) and gasreagent $\left(\mathrm{O}_{2}, \mathrm{~N}_{2}, \mathrm{NH}_{3}, \mathrm{CH}_{4}\right)$ makes it possible to obtain the mixtures of the nano-crystalline oxides of different metals, oxcides, nitrides or carbide-nitride mixtures. Composition and the size of nano-particles can be controlled by change in pressure and composition of inert gas atmosphere and gas reagent, by power of laser pulse, temperature gradient during the cooling process. Two reasons for the fine powder to be more conductive can be taken into account. One of them is the strength of electric field increase due to the size and shape of the particles. Another one is the concentration of particles in the dust plasma track of "Impulsar" [5]. The content of nanoparticles in the sublimated substance usually composes $10 \%-15 \%$ of the mass. As the source of laser emission, high repetition rate $\mathrm{P}-\mathrm{P}$ laser should be used. The moving focusing system is started directly from the earth's surface with the aid of high repetition rate P-P laser emission with the energy sufficient for the medium breakdown in the focus of optical system (Figure 4).

As a result of laser radiation concentration in the relatively small volume, the breakdown of medium takes place. Air breakdown appears in the region of ionization, which applies to a certain distance following the moving focusing system, and the shock wave, which gives to a moving system additional momentum, leading to its acceleration. Upon the transfer of moving focusing system into rarefied layers of the atmosphere or open space, breakdown and appearing in this case shock wave will be achieved in the easily ionized substance, which the moving system has been supplied in advance. With the arrival of the moving focusing system up to the assigned distance or on reaching by the current conducting channel the energy receiver, it is necessary to place the electrode of high voltage source into the canal and to ensure the energy transfer process. The conductivity of channel proved to be sufficient for the discharge of capacitor bank to the ground just after reaching by the moving optical set of the grounded electrode, located at the final point of lift.

The results of dust plasma electrical breakdown tests are presented here without detailed explanation of physical matter of experimental arrangements. The most simple and effective dust plasma production methods were involved into our investigation. Best result of specific voltage for dust plasma breakdown was measured

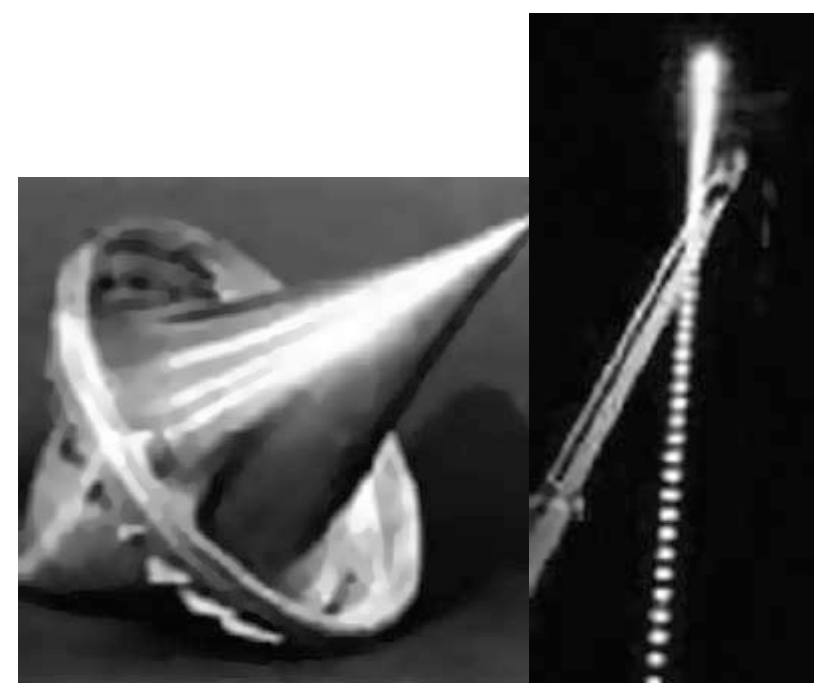

Figure 4. Launch of vehicle by low rate P-P laser (Rep. rate$20 \mathrm{~Hz}, \mathrm{P}=10 \mathrm{~kW})$.

for electrical $\mathrm{Cu}$ wire explosion approach. The results of dust plasma modeling are [6,7]:

1) P-P $\mathrm{CO}_{2}$ laser with train of pulses and total energy - 20 J. Dust particles size 50 - $250 \mathrm{~nm}$ and concentration $\mathrm{N}=10$ to the $10-12$ power.

For dust plasma produced by laser ablation: paraffin $\mathrm{wax}+$ graphite or permalloy powder $-300 \mathrm{v} / \mathrm{cm}$;

For nano-particles of $\mathrm{Al}, \mathrm{Fe}$, magnetoelectrics-350 $\mathrm{v} / \mathrm{cm}$;

2) $\mathrm{CO}_{2}$ single pulse laser channel, produced by conical optics - $100 \mathrm{ohm} / \mathrm{m}, 100 \mathrm{~V} / \mathrm{cm}$;

3) Long channel (filaments) produced by solid state laser- $0.1 \mathrm{Mohm} / \mathrm{m}, 7.5 \mathrm{kV} / \mathrm{cm}$;

4) Long channel produced by wire explosion-10 $\mathrm{ohm} / \mathrm{m}, 45$ - $50 \mathrm{~V} / \mathrm{cm}$;

5) Data for wire explosion channel, corresponding to the delays: $10 \mathrm{mks}$ - $50 \mathrm{mks}$ - $75 \mathrm{mks} ; 97 \mathrm{~V} / \mathrm{cm}-124$ $\mathrm{V} / \mathrm{cm}-166 \mathrm{~V} / \mathrm{cm}$.

It should be mentioned here that min. resistance value is very much dependable on cross-section of dust particles sheaf, concentration and size of particles, repetition rate of laser pulses, average power of laser, gas pressure, humidity and many other parameters are involved.

\section{CONCLUSIONS}

Powerful pulsed lasers are capable to create a large scale current conducting channels, which can be located at the arbitrary distances from the source of radiation. Channel with the length about hundred of meters can be achieved with low energy of single laser pulse. Beginning from the 70's, successful attempts of the problems: interception of lightning bolts and blocking of over voltage waves on the electric power lines were undertaken.

Successful developments of high repetition rate P-P 
powerful lasers technology and technology of "Impulsar" system make it possible to foresee the possibility of well conducting channels realization with the length up to several ten and hundreds of kilometers for the purpose of energy transfer for significant distances, creation of new and promising systems for the mastering of outer space power engineering and motivation for significant contribution to be done on that basis to an essential improvements of the global ecology of our planet.

\section{REFERENCES}

[1] Apollonov, V.V., Sorochenko, V.R. and Shakir, Yu.A. (2009) Experimental investigation of resonance single wire line of energy transfer. Report of GPI RAS.

[2] Teramobile: Site in INTERNET, French (CNRS)-German
(DFG) scientific research program funded by ANR: First mobile terawatt laser in the world for atmospheric studies.

[3] Apollonov, V.V. (2008) To the space by laser light. Vestnik of RANS 1.

[4] Apollonov, V.V. and Vagin, Yu.S. (2009) Conducting channel creation in a non-conductive media. Patent No. 2009118874.

[5] Apollonov, V.V. and Pletnev, N.V. Special features of conducting channel formation in atmosphere. In Press.

[6] Apollonov, V.V. and Pletnev, N.V. (2012) Formation of extended conducting channels in atmosphere. Quantum Electronics, 42, 130. doi:1070/QE2012v042n02ABEH014762

[7] Apollonov, V.V. and Pletnev, N.V. Formation of long directed breakdown channels in the atmosphere. In Press. 